\title{
Decreasing radiographs in neonates through targeted quality improvement interventions
}

\author{
Patrick Motz $\mathbb{1}^{1} \cdot$ Julie Do $^{1} \cdot$ Teresa $_{\text {Lam }}^{1} \cdot$ Robert M. DiBlasi ${ }^{2} \cdot$ Tim Fang $^{2} \cdot$ Karen Kelly $^{2} \cdot$ Robert DiGeronimo $^{1} \cdot$ \\ Zeenia C. Billimoria ${ }^{1}$
}

Received: 25 April 2019 / Revised: 14 November 2019 / Accepted: 18 November 2019 / Published online: 16 December 2019

(c) The Author(s), under exclusive licence to Springer Nature America, Inc. 2019

\begin{abstract}
Objective Our aim was to decrease radiograph use for monitoring placement of peripherally inserted central catheters (PICC) and endotracheal tubes (ETT) in neonates admitted to the neonatal intensive care unit (NICU) by $20 \%$ from November 2017 to November 2018.

Study design We carried out three Plan-Do-Study-Act (PDSA) cycles: (1) implementation of a radiograph protocol emphasizing ideal patient positioning, standard radiograph views and frequency, (2) standardizing ETT depth using the NRP guidelines, and (3) implementation of an institution specific ETT depth guideline.

Results The pre-intervention radiographs per PICC day was 0.86 versus a post-intervention value of $0.46(P=0.004)$. The pre-intervention radiographs per ETT day was 1.45 versus a post-intervention value of $1.07(P=0.002)$.

Conclusions Our multidisciplinary NICU team performed a QI project, which resulted in more than a $20 \%$ decrease in the number of radiographs used for monitoring placement of PICCs and ETTs.
\end{abstract}

\section{Introduction}

Critically ill neonates often require peripherally inserted central catheters (PICC) and endotracheal tubes (ETT) during their neonatal intensive care unit (NICU) stay $[1,2]$. PICCs allow for adequate drug and nutrition delivery intravenously in neonates unable to receive these enterally [3]. ETTs allow for mechanical ventilation to support neonates with respiratory failure [4].

While both PICCs and ETTs are commonly used in neonates, when malpositioned, they are associated with an increased risk of complications. Known PICC complications related to malposition include thrombosis, arrhyth-

Supplementary information The online version of this article (https:// doi.org/10.1038/s41372-019-0565-9) contains supplementary material, which is available to authorized users.

Zeenia C. Billimoria

zeenia.billimoria@seattlechildrens.org

1 University of Washington School of Medicine, Seattle, WA, USA

2 Seattle Children's Hospital and Research Institute, Seattle, WA, USA mia, and migration into pleural, pericardial or peritoneal spaces [2, 5-8]. Unplanned extubations associated with ETT malposition often require emergent intubations, which can increase risk of airway trauma, subglottic stenosis and ventilator associated pneumonia [4].

To prevent PICC and ETT malposition, they are typically monitored with serial imaging to prevent complications associated with malposition [9-11]. Unfortunately, there are no agreed upon consensus guidelines for NICUs regarding how often this should be done. A review of the literature by Nadroo showed significant variation in modalities used and interobserver reliability to identify PICC tip position [12]. Supine radiographs are most commonly used but are fraught with interpretation challenges due to arm or leg positioning and can result in repeat exposure to radiation $[12,13]$. ETTs are most commonly monitored by chest radiographs; however ultrasound has also been used $[9,10]$. Additionally, neck position can greatly impact ETT position on supine chest radiograph [14].

Exposure to cumulative doses of radiation increases the risk of malignancy [15]. Several studies have raised concerns about the relatively high rate of radiographs ordered on NICU patients putting them at long-term risk [16-18]. Overall radiation exposure from radiographs in neonates may be lower than natural background radiation and the risk 
of fatal malignancy is unclear [19]. Limiting unnecessary radiograph exposure in neonates is important to decrease long-term complications in this population at risk for several comorbidities.

Radiograph exposure in our neonates with PICC and ETT was high due to repeat radiographs as a result of variable positioning of patient. A review of our radiograph exposure showed an average of 0.86 radiographs per PICC line day or 810 radiographs for 120 patients with PICC lines, and 1.45 radiographs per ETT tube day or 3329 radiographs for 120 patients with ETTs. We identified an opportunity in our own practice to potentially improve outcomes and decrease the frequency of radiographs to monitor PICC and ETT position in neonates. Using the model for improvement quality improvement (QI) methodology, we implemented a series of PDSA cycles to decrease the number of radiographs performed on NICU patients with PICC and ETT.

\section{Methods}

\section{Setting}

The QI intervention was conducted from November 2017 to November 2018 in a Level IV NICU at Seattle Children's Hospital. Our NICU is a busy regional referral center for a five-state area, which includes Washington, Wyoming, Alaska, Montana, and Idaho serving neonates with a wide range of surgical and complex medical needs. The NICU cares for neonates ranging from 22 weeks gestational age to post term. All patients are outborn and referred in from other medical centers or internally from our emergency department.

In our NICU, PICCs are placed by a team of PICC nurses and neonatal nurse practitioners. Their first few PICC placements are proctored by senior PICC nurses. PICC depth is calculated at the time of placement. Upper extremity PICCs are measured from the point of insertion, along the proposed vein track to the third intercostal space. Lower extremity PICCs are measured from the insertion site up the leg to the groin and then above the level of the diaphragm/xiphoid. A system for periodic re-certification for PICC placement currently does not exist. ETT depth on initial placement is determined by the proceduralist. Commonly used depth guideline is weight in kilogram +6 rule, while others used direct visualization of the vocal cords. Both are confirmed with auscultation of equal breath sounds bilaterally. Monitoring of PICC and ETT position using radiographs is based on provider preference or when inadvertent change in position is suspected based on exam findings prior to implementation of the QI initiative.

\section{Ql initiative}

A multidisciplinary NICU team including neonatologists, neonatology fellows, respiratory therapists, and nurses met with the goal to standardize radiographic monitoring of neonates with PICCs and/or ETTs. We performed a thorough literature review and examined disparities in our current unit practice. Three key drivers to effect change were identified: (1) knowledge of ideal PICC and ETT position, (2) placement and care of PICC and ETT, (3) provider and staff engagement (Fig. 1). Pre-intervention clinical metrics, patient characteristics, and number of radiographs obtained to evaluate PICC and ETT position from January to October 2017 were obtained by review of the electronic medical record (EMR). Our study included a convenience sample of patients with PICC and ETTs. Each month we generated a list of eligible neonates with PICCs and/or ETTs. The list was randomly divided and sent out to the team involved in data collection. We set a goal minimum of 10 neonates audited per month. Data was collected in a REDCap database. Audits included number of radiographs ordered, manipulations of PICCs and ETTs and the positioning of the neonates during the radiographs. Manipulation of PICC and ETTs was defined as anytime a PICC or ETT was moved if it was in improper position as determined by the provider. There were no set standards for PICC and ETT position in the pre-intervention period. Compliance to the positioning protocol was assessed by determining if the positioning protocol was followed, by reviewing the radiographs of the study population. It was recorded as all or none. So, if the positioning was mostly correct, but not completely protocol compliant, it was recorded as non-compliant. A radiograph obtained in an infant with both a PICC and ETT was counted as one film for each device as each device may be manipulated with a radiograph and it would be challenging to separate them.

We carried out three Plan-Do-Study-Act (PDSA) cycles to achieve our specific, measurable, achievable, relevant and time bound (SMART) aim. In PDSA cycle 1, we implemented a radiograph protocol (PDSA cycle 1-13 November 2017 to April 2018). In this protocol (Supplementary Table 1), we implemented standard positioning for radiograph of neonates with PICC and ETT, standard radiograph views and frequency of radiographs for monitoring position. Protocol implementation involved training key staff including radiograph technologists, bedside nurses, respiratory therapists, fellows, and attendings. Training was done via nursing staff in-service and communication with staff in the monthly newsletters. Weekly radiographs for PICC lines was operationalized by adding a bullet point to our post rounding safety checklist on each patient in the unit. This post rounds safety checklist was reviewed by the team after each patient presentation. 


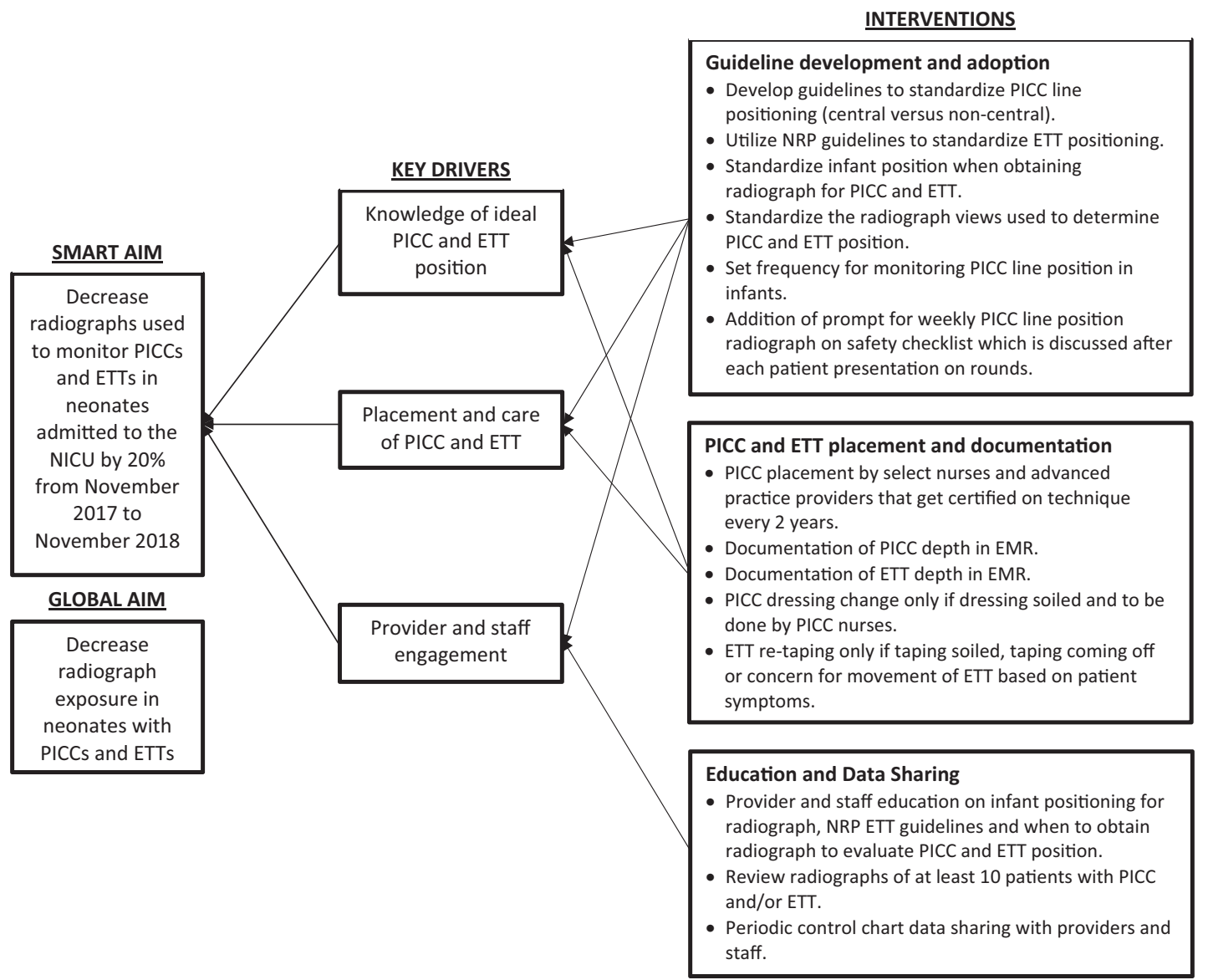

Fig. 1 Key driver diagram.

In PDSA cycle 2 (May 2018-August 2018), we implemented a new weight-based ETT depth guideline based on the 2016 Neonatal Resuscitation Program (NRP) recommendations since we found that $43 \%$ of ETT manipulations pre-intervention were due to malposition following initial placement. The newly implemented depth guideline was used for initial placement of ETTs only (Supplementary Fig. 1). Radiographic confirmation of ETT depth was considered the gold standard for determining optimal position.

In PDSA cycle 3 (September 2018-November 2018), we further revised our recently implemented ETT guideline from PDSA cycle 2 to better optimize ETT position, especially for those neonates $\geq 3 \mathrm{~kg}$ as we found a higher incidence of malposition (ETT at T1 or higher) in this subpopulation of patients. Additional changes were added to better optimize initial depth of ETT placement for smaller neonates under $3 \mathrm{~kg}$ as well as adding recommendations for those patients over $4 \mathrm{~kg}$ who also are routinely cared for in our NICU (Supplementary Fig. 2).

Simultaneously, education occurred around care of PICC dressing and ETT taping. PICC dressings were changed only if the dressing was visibly soiled or was coming undone exposing the entry point of the PICC line. PICC dressings were changed only by PICC nurses for consistency and prevention of line associated infections. ETT re-taping was done only if its integrity was questionable putting the patient at risk of unplanned extubation. In our unit, all ETTs are secured with a modified umbilical clamp called the "bone". This practice has not changed in several years. Documentation of PICC and ETT depth in the electronic medical record was standardized.

NICU staff and providers were educated prior to implementation of each PDSA cycle and results were shared with them periodically.

\section{Outcome measures}

Our primary outcome was the number of radiographs performed in neonates with a PICC and/or ETT per day while they were in place for the convenience sample we audited each month. Secondary outcomes included the overall number of PICC and ETT manipulations as well as radiograph charges per day. Compliance with the radiograph protocol was evaluated by conducting monthly audits of ordered patient radiographs. We additionally tracked 
Table 1 Baseline characteristics of neonates with PICC and/or ETT.

\begin{tabular}{|c|c|c|c|c|}
\hline & Overall $(n=260)$ & $\begin{array}{l}\text { Pre-intervention } \\
(n=120)\end{array}$ & $\begin{array}{l}\text { Post-intervention } \\
(n=140)\end{array}$ & $P$ \\
\hline Gestational age when PICC or ETT placed (weeks) $($ mean \pm SD) & $34.1 \pm 5.3$ & $34.0 \pm 5.3$ & $34.2 \pm 4.9$ & 0.89 \\
\hline Gestational age $<28$ weeks $(\%)$ & 29.5 & 29.4 & 29.6 & 0.96 \\
\hline Weight when PICC placed $(\mathrm{g})($ mean \pm SD) & $2700.3 \pm 1092.0$ & $2670.4 \pm 1092.9$ & $2730.3 \pm 1208.6$ & 0.72 \\
\hline Weight when ETT placed $(\mathrm{g})($ mean $\pm \mathrm{SD})$ & $2615.8 \pm 1449.5$ & $2589.0 \pm 1294.5$ & $2642.6 \pm 1449.9$ & 0.93 \\
\hline Male gender $(\%)$ & 58.4 & 58.3 & 58.6 & 0.92 \\
\hline PICC radiographs & 1266 & 810 & 456 & 0.002 \\
\hline ETT radiographs & 5503 & 3329 & 2174 & 0.003 \\
\hline
\end{tabular}

unplanned extubations and PICC complications as balancing measures. PICC complications included thrombosis, line breakage, CLABSI, and pericardial effusion.

\section{Inclusion/exclusion criteria}

Neonates were eligible if they were admitted to the NICU and had a PICC and/or ETT in place. This included new admits with ETTs/PICCs in place and neonates who had them placed during their stay in our NICU. Neonates were excluded if they did not have a PICC and/or ETT in place during their admission or if they required extracorporeal membrane oxygenation.

\section{Data analysis}

We used QI methodology with monthly run charts to track our outcomes. The U-chart was used as the primary statistical process control (SPC) chart to determine the impact of our interventions. We used rule-based conventions to identify special cause variation [20].

Demographic, clinical, and laboratory characteristics of the neonates, including birth gestational age, corrected gestational age, gender, diagnosis, weight when PICC and/ or ETT were placed, site of PICC placement, size of the PICC, ETT depth, and ETT size were collected. Data were summarized using means and standard deviations for continuous variables and counts and proportions for categorical variables. We used student's $T$-test to determine if the preintervention group was different from the post-intervention group. Statistical analyses were performed using $\mathrm{R}$ Version 1.14.4 (Vienna, Austria). $P$ value $<0.05$ was considered statistically significant.

\section{Ethical considerations}

This QI project was approved by the Seattle Children's Hospital Institutional Review Board. We implemented an evidence based radiographic monitoring protocol for neonates with PICCs and/or ETTs in our NICU. No protected health information was disclosed as part of this project.

\section{Results}

We started our QI process on 14 November 2017 and continued until 14 November 2018. Baseline data on demographics, number of radiographs for PICC and/or ETT placement was gathered from 1 January 2017 until 13 November 2018. There were no differences in gestational age at PICC/ETT placement, gender, weight at the time of PICC and/or ETT placement between pre- and postintervention groups and proportion of $<28$ week neonates (Table 1). Compliance with the new radiograph protocol was $88 \%$ during the study period.

Prior to implementation of our protocol, average radiographs ordered per PICC line day were 0.86 and after protocol implementation average radiographs ordered per PICC line day decreased to $0.46(P=0.003)$. We also observed a decrease in radiographs for ETT; pre-protocol average radiographs per ETT day were 1.45 and postprotocol average radiographs per ETT day were $1.06(P=$ 0.002) (Table 2). Rates of radiograph exposure for PICCs were lower in the post-intervention period. We identified special cause variation of more than eight consecutive data points below the centerline. This established a new centerline for number of radiographs per PICC day (Fig. 2). Rates of radiograph exposure were lower in the post-intervention period for ETTs. We identified special cause variation of more than 8 consecutive data points below the centerline. This established a new centerline for number of radiographs per ETT day (Fig. 3).

Average manipulations per PICC line day was 0.18 prior to the QI interventions. Average manipulations per PICC line day decreased to 0.06 post-intervention $(P=0.01)$. ETT manipulations also decreased from 0.33 to 0.19 after post-intervention $(P=0.02$ ) (Table 2 ). We identified special 
cause variation in our data for both PICC and ETT manipulations. This was demonstrated by more than 8 data points below the centerline for both PICC and ETT manipulations. (Supplementary Fig. 3 and 4).

Radiograph charges in the pre-intervention group per PICC day were $\$ 353.77$ and in the post-intervention group, they were $\$ 187.73(P=0.001)$. Charges saved for our sample population between the pre- and post-intervention group was $\$ 182,982.92$. We did not collect data on total PICC line days for all neonates in the NICU during our study period, but instead only for those sampled in the

Table 2 Pre- and post-intervention primary and secondary outcome measures.

\begin{tabular}{|c|c|c|c|}
\hline & $\begin{array}{l}\text { Pre-Intervention } \\
(n=120)\end{array}$ & $\begin{array}{l}\text { Post-intervention } \\
(n=140)\end{array}$ & $P$ \\
\hline \multicolumn{4}{|l|}{ Average radiographs ordered } \\
\hline per PICC line day & 0.86 & 0.46 & 0.003 \\
\hline per ETT day & 1.45 & 1.06 & 0.002 \\
\hline \multicolumn{4}{|l|}{ Average manipulations } \\
\hline per PICC line day & 0.18 & 0.06 & 0.01 \\
\hline per ETT day & 0.33 & 0.19 & 0.02 \\
\hline \multicolumn{4}{|l|}{ Radiograph charges $(\$)$} \\
\hline per PICC day & 353.77 & 187.73 & 0.01 \\
\hline per ETT day & 593.46 & 432.70 & 0.01 \\
\hline \multicolumn{4}{|l|}{ Balancing measures } \\
\hline $\begin{array}{l}\text { PICC complications } \\
\text { per } 1000 \text { central line days }\end{array}$ & 30 & 30 & 0.92 \\
\hline $\begin{array}{l}\text { Unplanned extubations } \\
\text { per } 100 \text { ventilator days }\end{array}$ & 0.3 & 0.3 & 0.93 \\
\hline
\end{tabular}

study. Therefore, we were unable to extrapolate charges saved for all NICU patients with PICCs. Radiograph charges for our sample population in the pre-intervention group per ETT day were $\$ 593.46$ and in the post-intervention group, they were $\$ 432.70(P=0.003)$. Charges saved between the pre- and post-intervention group was $\$ 186,958.03$. If we assume the effect of our intervention is the same for the group of patients with ETTs not collected in the data set (data on ventilator days obtained from another QI project) during our intervention time, we extrapolated an estimated savings of \$718,253.19 (Table 2).

For our balancing measures, we compared unplanned extubations and PICC complications. There were no significant differences amongst our balancing measures between the pre-intervention and post-intervention groups.

\section{Discussion}

As part of a SMART aim directed QI project for our NICU, we successfully implemented a unit based radiograph protocol and ETT depth guideline for neonates with PICCs and/or ETTs that standardized patient positioning, radiograph views, frequency of radiographic monitoring, and depth of ETT insertion. In our study population, we were able to show a significant decrease in the number of radiographs ordered for neonates with PICCs and/or ETTs. We also demonstrated a decrease in PICC line and ETT manipulations and radiograph charges during the study period without increasing unplanned extubations or PICC line complications.

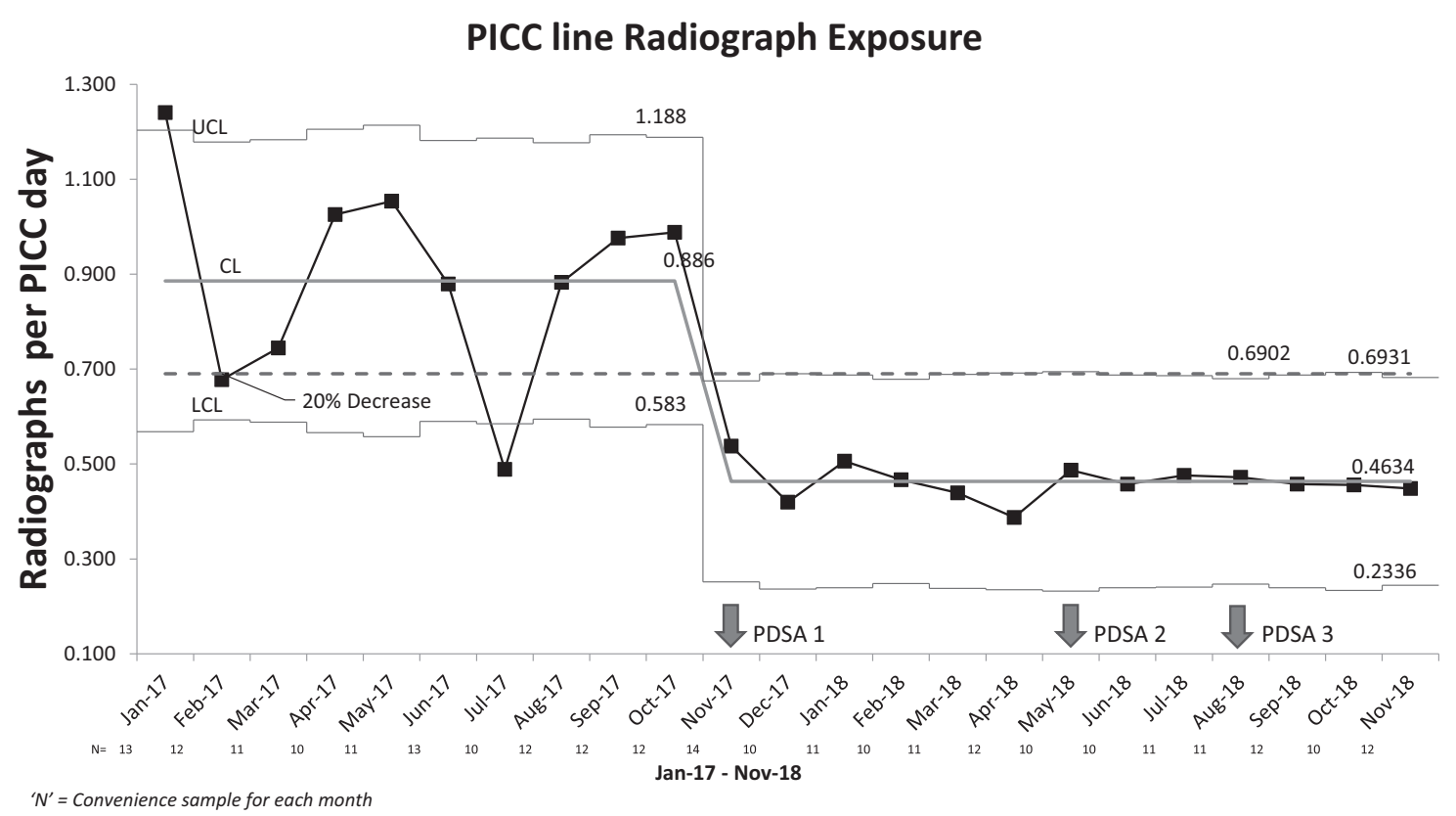

Fig. 2 PICC line radiograph exposure. 


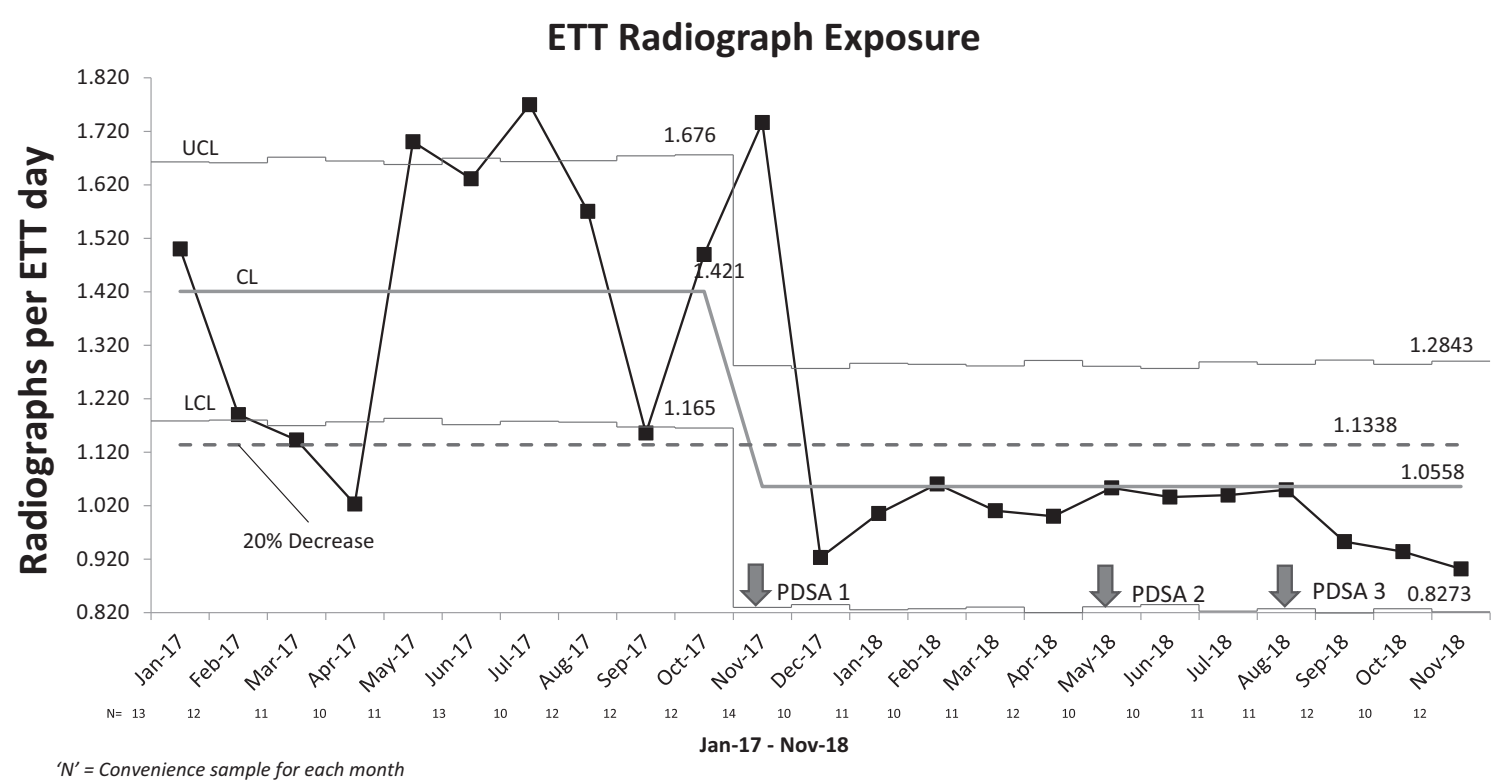

Fig. 3 ETT radiograph exposure.

Various studies have evaluated positioning of patients during radiographs with PICCs and ETTs [10, 11, 14]. However, studies are lacking on ideal frequency of monitoring PICCs and ETTs. A quality improvement initiative at Children's National Health System NICU to limit frequency of radiographs monitoring ETT position to twice weekly, yielded a lower unplanned extubation rate, less radiation exposure and decreased charges in imaging costs over a 6 month period [20,21]. Radiographic monitoring of PICCs and ETTs has been thoroughly evaluated and is currently considered the gold standard [2, 3]. However, there is emerging evidence advocating for the use of bedside ultrasound technology to monitor PICC positioning in lieu of frequent radiographs [22]. Future QI projects will likely focus on the utility of point of care ultrasound to assess ETT and central line placement versus routine radiograph monitoring.

Our second and third PDSA cycles focused on ETT depth. Recent research has proposed weight and gestational age-based guidelines, and NRP has adopted a weight-based guideline [23]. While weight-based NRP guidelines for ETT depth seemingly work well in preterm smaller birth weight neonates, there is emerging evidence that it may underestimate ETT depth in larger older neonates [24]. Consistent with these concerns, we found in our study during PDSA cycle 2 that using NRP guidelines for initial ETT placement resulted in consistently high positioning in neonates over $3 \mathrm{~kg}$. Given this finding, we amended our guideline to adopt the weight in kilogram $+6 \mathrm{~cm}$ rule for neonates $3 \mathrm{~kg}$ or above. Our third PDSA cycle subsequently demonstrated that this modified guideline resulted in more accurate initial ETT placement and we have integrated it into routine NICU practice.
Our study has several strengths. Our sample size of 260 patients was robust, we were able to show special cause variation for our interventions, and we did not show an increase in adverse events. Some limitations of this study are related to the nature of quality improvement projects. We did not randomize our patients and the NICU providers and staff were not blinded to the results. This increases the risk of intention and chronology bias. We also picked a convenience sample of 10 neonates per month, albeit randomly assigned, which may also have contributed to selection bias. Patient disease and severity could potentially confound our results. However, we selected patients randomly each month which should decrease this effect. It would be interesting to study the number of initial radiographs as a possible outcome for future studies. We could not parse this out from the data we collected. Additionally, our QI intervention was designed specifically for our large regional referral NICU and the successful outcomes we found may not necessarily be generalizable to other NICUs.

In conclusion, for this study, we implemented a QI focused radiograph protocol and ETT depth guideline for neonates with PICCs and ETTs that standardized patient positioning, radiograph views, frequency of radiographic monitoring, and depth of ETT insertion. Our combined interventions decreased the number of radiographs performed in neonates with PICCs and/or ETTs, decreased manipulations of PICCs and ETTs, and decreased radiograph charges. We feel this study highlights the need for and efficacy of standardizing radiographic monitoring of PICCs and ETTs.

Acknowledgements We would like to acknowledge Joseph Zimmerman RT, Jami "Mari" Clarkmoore RN for their help with data collection on this project. 


\section{Compliance with ethical standards}

Conflict of interest RMDiBlasi is on the Speaker's Bureau for Draeger. He has also received research funding and/or speaker honoraria from Aerogen Pharma, Vero Biopharma, Neotech and Vapotherm. The other authors have nothing to disclose.

Publisher's note Springer Nature remains neutral with regard to jurisdictional claims in published maps and institutional affiliations.

\section{References}

1. Ainsworth S, McGuire W. Peripherally inserted central catheters vs peripheral cannulas for delivering parenteral nutrition in neonates. J Am Med Assoc. 2016;315:2612-3. https://doi.org/10. 1001/jama.2016.7020

2. Hatch LD, Grubb PH, Lea AS, Walsh WF, Markham MH, Whitney GM, Ely EW. Endotracheal intubation in neonates: a prospective study of adverse safety events in 162 infants. J Pediatrics. 2016. https://doi.org/10.1016/j.jpeds.2015.09.077

3. Jain A, Deshpande P, Shah P. Peripherally inserted central catheter tip position and risk of associated complications in neonates. J Perinatol. 2012;33:307-12. https://doi.org/10.1038/jp. 2012.112

4. Sawyer T, Foglia E, Hatch LD, Moussa A, Ades A, Johnston L, Nishisaki A. Improving neonatal intubation safety: a journey of a thousand miles. J Neonatal-Perinat Med. 2017;10:125-31. https:// doi.org/10.3233/npm-171686

5. Milstone, AM, Reich, NG, Advani, S, Yuan, G, Bryant, K, Coffin $\mathrm{S}$, et al. Catheter dwell time and CLABSIs in neonates with PICCs: a multicenter cohort study. Pediatrics. 2013. https://doi. org/10.1542/peds.2013-1645d

6. Park CK, Paes Ba, Nagel K, Chan AK, Murthy P. Neonatal central venous catheter thrombosis: diagnosis, management and outcome. Blood Coagul Fibrinolysis 2014;25:97-106. https://doi.org/10. 1097/MBC.0b013e328364f9b0

7. Katheria AC, Fleming SE, Kim JH. A randomized controlled trial of ultrasound-guided peripherally inserted central catheters compared with standard radiograph in neonates. J Perinatol 2013;33:791-4. https://doi.org/10.1038/jp.2013.58

8. Arnts IJJ, Bullens LM, Groenewoud JMM, Liem KD. Comparison of complication rates between umbilical and peripherally inserted central venous catheters in newborns. J Obstet Gynecol Neonatal Nurs 2014;43:205-15. https://doi.org/10.1111/1552-6909.12278

9. Zaytseva A, Kurepa D, Ahn S, \& Weinberger B. Determination of optimal endotracheal tube tip depth from the gum in neonates by Radiograph and ultrasound. J Matern Fetal Neonatal Med. 2018. https://doi.org/10.1080/14767058.2018.1538350

10. Sharma D, Tabatabaii SA, \& Farahbakhsh N. Role of ultrasound in confirmation of endotracheal tube in neonates: a review. $\mathrm{J}$
Matern Fetal Neonatal Med. 2017. https://doi.org/10.1080/ 14767058.2017.1403581

11. Sharma D, Farahbakhsh N, \& Tabatabaii SA .Role of ultrasound for central catheter tip localization in neonates: a review of the current evidence. J Matern Fetal Neonatal Med. 2018. https://doi. org/10.1080/14767058.2018.1437135

12. Nadroo A, Lin J, Green R, Magid M, Holzman I. Death as a complication of peripherally inserted central catheters in neonates. J Pediatrics. 2001;138:599-601. https://doi.org/10.1067/mpd. 2001.111823

13. Nadroo A, Glass R, Lin J, Green RS, Holzman IR. Changes in upper extremity position cause migration of peripherally inserted central catheters in neonates. Pediatrics. 2002. http://pediatrics.aa ppublications.org/content/110/1/131.long

14. Olufolabi A, Charlton G, Spargo P. Effect of head posture on tracheal tube position in children. Anaesthesia 2004;59:1069-72. https://doi.org/10.1111/j.1365-2044.2004.03963.x

15. González B, Darby S. Risk of cancer from diagnostic X-rays: estimates for the UK and 14 other countries. Lancet 2004;363:345-51.

16. Lai $\mathrm{T}, \mathrm{Barer} \mathrm{C}$. Iatrogenic environmental hazards in the neonatal intensive care unit. Clin Perinatol. 2008;35:163-81.

17. Yu C. Radiation safety in the neonatal intensive care unit: too little or too much concern? Pediatr Neonatol. 2010;51:311-9. https:// doi.org/10.1016/S1875-9572(10)60061-7

18. Iyer N, Baumann A, Rzeszotarski M, Ferguson R, Mhanna M. Radiation exposure in extremely low birth weight infants during their neonatal intensive care unit stay. World $J$ Pediatr 2013;9:175-8. https://doi.org/10.1007/s12519-013-0417-1

19. Puch-Kapst K, Juran R, Stoever B, Wauer R. Radiation exposure in 212 very low and extremely low birth weight infants. Pediatrics. 2009;124:1556-64.

20. Montgomery D. Introduction to statistical quality control. New York: Wiley;2013.

21. Ridore M, Pastor W, Bulas D, McKenney S, Soghier L, Short B. Initiation and Compliance with a Chest Radiograph (CXR) Reduction Protocol in the Children's National Medical Center Neonatal Intensive Care Unit (NICU). Vol 142/Issue 1 Meeting Abstract. Section on Neonatal-Perinatal Medicine Program: Day 1. 2018. http://pediatrics.aappublications.org/content/142/1_MeetingAbstra $\mathrm{ct} / 221$

22. Motz P, Arnim A, Likes M, Chabra S, Traudt C, Iyer R, Dighe M. Limited ultrasound protocol for upper extremity peripherally inserted central catheter monitoring: a pilot study in the neonatal intensive care unit. J Ultrasound Med. 2018. https://doi.org/10. $1002 /$ jum. 14816

23. Kempley S, Moreiras J, Petrone F. Endotracheal tube length for neonatal intubation. Resuscitation 2008;77:369-73. https://doi. org/10.1016/j.resuscitation.2008.02.002

24. Chung H, Lee W, Chen H. Reexamining the ideal depth of endotracheal tube in neonates. Pediatr Neonatol. 2018;59:258-62. https://doi.org/10.1016/j.pedneo.2017.10.001 